\title{
SINGLE INTERCONNECTION OF KURAMOTO COUPLED OSCILLATORS
}

\author{
Pablo Monzón ${ }^{*}, 1$ Eduardo Canale ${ }^{* *}$ \\ * Instituto de Ingeniería Eléctrica \\ ** Instituto de Matemática y Estadística (IMERL), \\ Facultad de Ingeniería, Montevideo, Uruguay
}

\begin{abstract}
In this work, we analyze the almost global synchronization property of sinusoidally coupled oscillators. In contrast with previous works, we introduce an approach that uses the strong basic facts of algebraic graph theory to prove dynamical properties of the standard symmetric Kuramoto model. We show how we can interconnect two (or several) systems via bridges, keeping the almost global synchronization. These ideas can be used to explore other kinds of interconnections. Copyright (c) IFAC 2007
\end{abstract}

Keywords: Almost global stability, Kuramoto coupled oscillators, nonlinear systems

\section{INTRODUCTION}

A few decades ago, Y. Kuramoto introduced a mathematical model of weakly coupled oscillators that gave a formal framework to some of the works of A.T. Winfree on biological clocks (Kuramoto, 1975; Kuramoto, 1984; Winfree, 1980). The model proposes the idea that several oscillators can interact in a way such that the individual oscillation properties change, in order to achieve a global behavior for the interconnected system. The Kuramoto model serves as a good representation of many systems in several contexts such a biology, engineering, physics, mechanics, etc. (Ermentrout, 1985; York, 1993; Strogatz, 1994; Strogatz, 2000; Dussopt, 1999; Rogge, 2004; Marshall, 2004; Moshtagh and Jadbabaie and Daniilidis, 2005).

Recently, many works on the control community have focused on the analysis of the Kuramoto model, specially the one with sinusoidal coupling. The consensus or collective synchronization of

\footnotetext{
1 Partially supported by CSIC-Udelar
}

the individuals is particularly important in many applications that need to represent coordination, cooperation, emerging behavior, etc. Local stability properties of the consensus have been initially explored in (Jadbabaie, 2004), while global or almost global dynamical properties were studied in (Monzón and Paganini, 2005; Monzón, 2006; Monzón and Paganini, 2006). In these works, the relevance of the underlying graph describing the interconnection of the system was hinted. In the present article, we go deeper on the analysis of the relationships between the dynamical properties of the system and the algebraic properties of the interconnection graph, exploiting the strong algebraic structure that every graph has.

In Section 2 we quickly review the relevant aspects of the algebraic graph theory. After that, we summarize the main results of different previous works on the analysis of Kuramoto coupled oscillators. Section 4 contains the contributions of this article, showing how we can interconnect synchronized systems keeping the synchronization property and introducing an analysis methodology. Finally, we present come conclusions. 


\section{ALGEBRAIC GRAPH THEORY}

In this Section, we review the basic facts on algebraic graph theory that will be used along the article. A more detailed introduction to this theory can be found in (Biggs, 1993; Cvetkovic and Doob and Sachs, 1979). A graph consists in a set of $n$ nodes or vertices $\mathcal{V}=\left\{v_{1}, \ldots, v_{n}\right\}$ and a set of $m$ links or edges $\mathcal{E}=\left\{e_{1}, \ldots, e_{m}\right\}$ that describes how the nodes are related to each other. We say that two nodes are neighbors or adjacent if there is a link in $\mathcal{E}$ between them. The graph is directed if every link has a starting node and a final node. The topology of a directed graph may be described by the incidence matrix $B$ with $n$ rows and $m$ columns:

$$
B_{i j}=\left\{\begin{array}{cl}
1 & \text { if edge } j \text { reaches node } i \\
-1 & \text { if edge } j \text { leaves node } i \\
0 & \text { otherwise }
\end{array}\right.
$$

The rank $r$ of $B$ is also called the rank of the graph. Observe that ${ }^{2} B^{T} \mathbf{1}_{n}=0$. By removing a link, say $e_{k}$, we obtain a new graph $G_{k}$ and we use the notation $G_{k}=G \backslash\left\{e_{k}\right\}$. If we split $G$ into two disjoints subsets $G_{1}$ and $G_{2}$, we define a cut of $G$ as the sets of links of $\mathcal{E}$ with initial or endings nodes in $G_{1}$ or $G_{2}$. Every graph has associated some vector spaces that lead to an algebraic and spectral graph theory. The cycle space of the graph $G$ is the kernel of the matrix $B$ and it is related with the cycles (non-intersecting closed paths) of the graph. Its dimension is equal to $m-$ $r$. The cut space is the orthogonal complement of the cycle space, with the euclidean inner product. Its dimension is equal to $r$. Given a cycle (cut) of $G$, there is a systematic way for constructing a vector in the cycle (cut) space. This will be used in Proposition 4.2.

\section{ALMOST GLOBAL SYNCHRONIZATION}

The state of an oscillator can be described by its phase angle $\theta$. As is explained in (Jadbabaie, 2004), when we have $n$ oscillators coupled in a sinusoidal way, the expression

$$
\dot{\theta}_{i}=\sum_{j \in \mathcal{N}_{i}} \sin \left(\theta_{j}-\theta_{i}\right) \quad i=1, \ldots, n
$$

describes the system, where $\mathcal{N}_{i}$ is the set of neighbors of agent $i$. Each phase $\theta_{i}$ belongs to the interval $[0,2 \pi)$, so the system evolves on the compact $n$-dimensional torus $\mathcal{T}^{n}$. The value of a phase must be considered modulo $2 \pi$. Consider the graph $G$, with nodes $\left\{v_{1}, \ldots, v_{n}\right\}$ and edges $\left\{e_{1}, \ldots, e_{m}\right\}$, that describes how the individual oscillators, or agents, interact between each others. The node $v_{i}$ represents the $i$-th oscillator, with

2 By $\mathbf{1}_{p}$ we denote the column vector in $\mathbb{R}^{p}$ with all its components equal to 1 . phase $\theta_{i}$. Consider an arbitrary orientation of the links of $G$ and let the matrix $B$, with $n$ rows and $m$ columns, be an incidence matrix. We will work with symmetric interaction: if $i \in \mathcal{N}_{j}$ then $j \in \mathcal{N}_{i}$. In this case, the system can be compactly written as

$$
\dot{\theta}=-B \sin \left(B^{T} \bar{\theta}\right)
$$

Equation (1) does not depend on the choice of $B$ (Jadbabaie, 2004). As was done by Kuramoto, we may represent the agents as running points on a circumference or as unit phasors (Kuramoto, 1975; Monzón, 2006).

Since the system dynamic depends only on the phase differences, it is invariant under translations parallel to vector $\mathbf{1}_{n}$. We say the system synchronizes or reaches consensus if the individuals phases converge to a state where all the phases are identical. Of course, a consensus point is an equilibrium point of the system and actually we have a synchronization set, due to the invariance property. This also applies to every equilibrium point. We will also work with partial consensus equilibria, when most of the phases take the value 0 (taking a suitable reference) and the remaining phases take the value $\pi$. Other equilibria will be referred as non-synchronized. If $\bar{\theta}$ is an equilibrium point of (1) with underlying graph $G$, we will use the expression: $\bar{\theta}$ is an equilibrium of $G$.

We are concerned on whether or not all the trajectories converge to the synchronization set. Since the system has many equilibria, we can only expect that most of the trajectories presents this property. Following the ideas of (Rantzer, 2001), we say that the system has the almost global synchronization property if the set of trajectories that do not converge to the synchronization set has zero Lebesgue measure on $\mathcal{T}^{n}$. If the system is described by a graph $G$, we will shortly say the $G$ is a.g.s. In (Jadbabaie, 2004), it was proved that the synchronization set is locally stable. First results on almost global properties were presented in (Monzón and Paganini, 2005; Monzón and Paganini, 2006). These results were proved in two steps: firstly, using LaSalle's result on asymptotical behavior of trajectories in a compact invariant set, it was shown that the only $\omega$-limit sets are the equilibria of the system; secondly, Jacobian linearization was used to locally classify the equilibria (Khalil, 1996). At an equilibrium point $\bar{\theta}$, a first order approximation is given by the symmetric, $n \times n$, matrix

$$
A_{G}=-B \operatorname{diag}\left[\cos \left(B^{T} \bar{\theta}\right)\right] B^{T}
$$

Observe that $A_{G}$ always has a single zero eigenvalue with corresponding eigenvector $\mathbf{1}$, due to the invariance property of the system. 


\section{SYNCHRONIZING INTERCONNECTION}

Consider two graphs $G_{1}$ and $G_{2}$. An interconnection between them will be done putting links between one or more agents of both graphs. We first start with the addition of one single link. Consider the following general result.

Proposition 4.1. Let $G$ be a graph with nodes $\left\{v_{1}, \ldots, v_{n}\right\}, e_{k}$ be a link between $v_{i}$ and $v_{i+1}$ and $\bar{\theta}$ an equilibrium point of $G$. Consider the graph $G_{k}=G \backslash\left\{e_{k}\right\}$. Then, $\bar{\theta}$ is an equilibrium point of $G_{k}$ if and only if $\bar{\theta}_{i}=\bar{\theta}_{i+1}$ or $\bar{\theta}_{i}-\bar{\theta}_{i+1}=\pi$.

Proof: Let $B$ and $B_{k}$ be respectively the incidence matrices of $G$ and $G_{k}$. $B$ can be decomposed as

$$
B=\left[\begin{array}{c|c|c} 
& 0 & \\
\vdots & & \\
-1 & B_{2} \\
& 1 & \\
\vdots & &
\end{array}\right]
$$

The size of $B_{1}$ is $n \times(k-1)$ and the size of $B_{2}$ is $n \times(m-k)$. The $k$-th column of $B$ has 0 everywhere except at places $i$ and $i+1$. With this notation,

$$
B_{k}=\left[B_{1} \mid B_{2}\right]
$$

Then,

$$
B \sin \left(B^{T} \bar{\theta}\right)=B_{k} \sin \left(B_{k}^{T} \bar{\theta}\right)-\left[\begin{array}{c}
0 \\
\vdots \\
\sin \left(\bar{\theta}_{i+1}-\bar{\theta}_{i}\right) \\
\sin \left(\bar{\theta}_{i}-\bar{\theta}_{i+1}\right) \\
\vdots \\
0
\end{array}\right]
$$

Since $\bar{\theta}$ is an equilibrium point,

$$
B_{k} \sin \left(B_{k}^{T} \bar{\theta}\right)=\left[\begin{array}{c}
0 \\
\vdots \\
\sin \left(\bar{\theta}_{i+1}-\bar{\theta}_{i}\right) \\
\sin \left(\bar{\theta}_{i}-\bar{\theta}_{i+1}\right) \\
\vdots \\
0
\end{array}\right]
$$

It is clear that in order to have an equilibrium point of $G_{k}$, it must be true that $\bar{\theta}_{i}=\bar{\theta}_{i+1}$ or $\bar{\theta}_{i}-\bar{\theta}_{i+1}=\pi$.

When the phase difference between two agents is zero, we say that they are in phase, while they are in counterphase when the pase difference is $\pm \pi$. If we have a graph $G$ with an equilibrium point $\bar{\theta}$ with two agents in phase or in counterphase, if those agents are not related, we can add a link between them and $\bar{\theta}$ is an equilibrium of the new graph.

\subsection{Algebraic analysis}

The previous result is for a non particular link between $v_{i}$ and $v_{k}$. Now, consider the case when the $k$-th link $e_{k}$, joining nodes $v_{i}$ and $v_{i+1}$, is a bridge of $G$. This means that the graph $G_{k}=G \backslash$ $\left\{e_{k}\right\}$ is not connected. We denote by $G_{1}$ and $G_{2}$ the connected components of $G_{k}$. Observe that, starting with two graphs $G_{1}$ and $G_{2}$, we can interconnect them via a single link, in order to have a bigger graph $G$ with a bridge.

Proposition 4.2. Consider the graph $G$ with a bridge $e_{k}$ between nodes $v_{i}$ and $v_{i+1}$. Then, for every $\bar{\theta}$ equilibrium point of $G$, it must be true that the interconnection between $v_{i}$ and $v_{i+1}$ is in phase or in counterphase.

Proof: Define the vector $w \in \mathbb{R}^{m}$ as follows:

$$
w_{k}=\left\{\begin{array}{l}
1 \text { if } h=k \\
0 \text { if } h \neq k
\end{array}\right.
$$

This vector belongs to the cut space of $G$ (Biggs, 1993) and so, it is orthogonal to every vector in the cycle space. Then, for every $\bar{\theta}$ equilibrium point of (1), we have the identity

$$
w_{k}^{T} \cdot \sin \left(B^{T} \bar{\theta}\right)= \pm \sin \left(\bar{\theta}_{i}-\bar{\theta}_{i+1}\right)=0
$$

Then, for every $\bar{\theta}$ equilibrium point of $G$, the $k$-th component of vector $\sin \left(B^{T} \bar{\theta}\right)$ must vanish and the phase difference $\bar{\theta}_{i}-\bar{\theta}_{i+1}$ is either 0 or $\pi$.

Since $e_{k}$ joins the subgraphs $G_{1}$ and $G_{2}$, we say that at the equilibrium point $\bar{\theta}$, these subgraphs are connected in phase if $\bar{\theta}_{i}=\bar{\theta}_{i+1}$ and in counterphase if $\bar{\theta}_{i}=\bar{\theta}_{i+1} \pm \pi$. This general result imposes an important restriction to every bridge of a given graph.

Proposition 4.3. Consider the graph $G$ with a bridge $e_{k}$ joining the nodes $v_{i}$ and $v_{i+1}$. Then, if $\bar{\theta}=\left[\bar{\theta}_{1}^{T}, \bar{\theta}_{2}^{T}\right]^{T} \in \mathbb{R}^{n}$ is an equilibrium point of $G$, with $\bar{\theta}_{1} \in \mathbb{R}^{i}$ and $\bar{\theta}_{2} \in \mathbb{R}^{n-i}$, then $\bar{\theta}_{1}$ and $\bar{\theta}_{2}$ are equilibrium points of $G_{1}$ and $G_{2}$ respectively. Conversely, if $\bar{\theta}_{1}$ and $\bar{\theta}_{2}$ are equilibrium points of $G_{1}$ and $G_{2}$ respectively, there exists a real number $\alpha$ such that $\bar{\theta}=\left[\bar{\theta}_{1}^{T}, \bar{\theta}_{2}^{T}+\alpha \cdot \mathbf{1}_{\mathbf{n}-\mathbf{i}}{ }^{T}\right]^{T}$ is an equilibrium point of $G$.

Proof: Let $B$ be the incidence matrix of $G$. Since the link $e_{k}$ is the only connection between $G_{1} \mathrm{y}$ 
$G_{2}$, we can order the nodes and links such that $B$ has the following particular form:

$$
B=\left[\begin{array}{c|c|c} 
& 0 & \\
B_{1} & \vdots & \mathbf{0}_{\mathbf{i} \times(\mathbf{m}-\mathbf{k})} \\
& -1 & \\
\hline & 1 & \\
\mathbf{0}_{(\mathbf{n}-\mathbf{i}) \times(\mathbf{k}-\mathbf{1})} & \vdots & B_{2}
\end{array}\right]
$$

$B_{1}$ and $B_{2}$ are $i \times(k-1)$ and $(n-i) \times(m-k)$ matrices respectively. The $k$-th column of $B$ has -1 at the row $i$ and a 1 at the row $i+1$. Assume that $\bar{\theta}$ is an equilibrium point of $G$. Then, by Proposition 4.2 , the $k$-th component of $\sin \left(B^{T} \bar{\theta}\right)$ is 0 and a direct calculation of the product of $B \sin \left(B^{T} \bar{\theta}\right)$ gives that

$$
B \sin \left(B^{T} \bar{\theta}\right)=\left[\begin{array}{l}
B_{1} \sin \left(B_{1}^{T} \bar{\theta}_{1}\right) \\
B_{2} \sin \left(B_{2}^{T} \bar{\theta}_{2}\right)
\end{array}\right]
$$

and we have proved the first part of the Proposition.

Now, assume that $\bar{\theta}_{1}$ and $\bar{\theta}_{2}$ are equilibrium points of $G_{1}$ and $G_{2}$ respectively. Let $\alpha$ be a real number such that the last component of $\bar{\theta}_{1}$ is $\alpha$ plus the first component of $\bar{\theta}_{2}$. Due to the invariance of the system we have remarked on Section 1, the vector $\bar{\theta}_{2}+\alpha \mathbf{1}_{\mathbf{n}-\mathbf{i}}$ is also an equilibrium point of $G_{2}$. In this case, $G_{1}$ and $G_{2}$ have an in phase interconnection and we recover the identity (5) and $\bar{\theta}=\left[\bar{\theta}_{1}^{T}, \bar{\theta}_{2}^{T}+\alpha \mathbf{1}_{\mathbf{n}-\mathbf{i}}^{\mathbf{T}}\right]^{T}$ is an equilibrium point of $G$. We could also have defined $\alpha$ such that the interconnection was in counterphase.

\subsection{Stability analysis}

We will relate the stability properties of the graph $G$ with a bridge with the stability properties of the resulting subgraphs $G_{1}$ and $G_{2}$. Since every equilibrium of $G$ defines an equilibria for $G_{1}$ and $G_{2}$, we wonder whether or not the dynamic characteristics of these equilibria are or not the same. We will use Jacobian linearization. The zero eigenvalue is always present due to the invariance of the system by translations parallel to $\mathbf{1}_{\mathbf{n}}$. We always refer to the transversal stability of the equilibrium set. If the multiplicity of the zero eigenvalue is more than one, Jacobian linearization fails in classifying the equilibria. Due to space reasons, we present the study of this particular problem in a different article. So, in this work, we assume that we always have a single null eigenvalue.

Theorem 4.1. Consider the graph $G$, with a bridge $e_{k}$ joining the nodes $v_{i}$ and $v_{i+1}$, and let $G_{1}$ and
$G_{2}$ be the connected components of the graph $G_{k}$. Let $\bar{\theta}=\left[\bar{\theta}_{1}, \bar{\theta}_{2}\right]^{T} \in \mathbb{R}^{n}$ be an equilibrium point of $G$, with $\bar{\theta}_{1} \in \mathbb{R}^{i}$ and $\bar{\theta}_{2} \in \mathbb{R}^{n-i}$. Then, $\bar{\theta}$ is locally stable if and only if $\bar{\theta}_{1}$ and $\bar{\theta}_{2}$ are locally stable and $G_{1}$ and $G_{2}$ have an in phase interconnection.

Proof: Recall that the first order approximation of the system around an equilibrium point is given by

$$
A_{G}=-B \operatorname{diag}\left[\cos \left(B^{T} \bar{\theta}\right)\right] B^{T}
$$

Due to the bridge $e_{k}, \cos \left(\bar{\theta}_{i}-\bar{\theta}_{i+1}\right)= \pm 1$ and

$$
\cos \left(B^{T} \bar{\theta}\right)=\left[\begin{array}{c}
\cos \left(B_{1}^{T} \bar{\theta}_{1}\right) \\
\pm 1 \\
\cos \left(B_{2}^{T} \bar{\theta}_{2}\right)
\end{array}\right]
$$

We introduce the auxiliary block diagonal matrix

$$
C=\left[\begin{array}{l|rr|l}
\mathbf{0}_{(\mathbf{i}-\mathbf{1}) \times(\mathbf{i}-\mathbf{1})} & & & \\
\hline & 1 & -1 & \\
& -1 & 1 & \\
\hline & & \mathbf{0}_{(\mathbf{n}-\mathbf{i}-\mathbf{1}) \times(\mathbf{n}-\mathbf{i}-\mathbf{1})}
\end{array}\right]
$$

This matrix is symmetric and positive semidefinite, with a single eigenvalue 2 and the null eigenvalue with multiplicity $n-1$. A direct calculation gives

$$
A_{G}=\left[\begin{array}{l|l}
A_{G_{1}} & \mathbf{0}_{\mathbf{i} \times(\mathbf{n}-\mathbf{i})} \\
\hline \mathbf{0}_{(\mathbf{n}-\mathbf{i}) \times(\mathbf{i})} & A_{G_{2}}
\end{array}\right] \mp C
$$

The - and + signs correspond to an in phase and counterphase interconnection respectively.

First of all, we consider the case with $\bar{\theta}_{1}$ and $\bar{\theta}_{2}$ stable. Then, $A_{G_{1}}$ and $A_{G_{2}}$ are stable. So, for an in phase interconnection, $A_{G}$ is the sum of two semidefinite negative matrices (recall that the vectors parallel to $\mathbf{1}_{\mathbf{n}}$ are in the kernel of $A_{G}$ and also in the kernel of $C$ ). This proves the stability of $A_{G}$.

Assume now that we face an in counterphase interconnection. Then,

$$
A_{G}=\left[\begin{array}{l|l}
A_{G_{1}} & \mathbf{0}_{\mathbf{i} \times(\mathbf{n}-\mathbf{i})} \\
\hline \mathbf{0}_{(\mathbf{n}-\mathbf{i}) \times(\mathbf{i})} & A_{G_{2}}
\end{array}\right]+C
$$

Consider the vector

$$
v=\left[\begin{array}{c}
\mathbf{1}_{\mathbf{i}} \\
-\mathbf{1}_{\mathbf{n}-\mathbf{i}}
\end{array}\right]
$$

We have that

$$
\begin{gathered}
v^{T} A_{G} v=v^{T}\left(\left[\begin{array}{l|l}
A_{G_{1}} & \mathbf{0}_{\mathbf{i} \times(\mathbf{n}-\mathbf{i})} \\
\hline \mathbf{0}_{(\mathbf{n}-\mathbf{i}) \times(\mathbf{i})} & A_{G_{2}}
\end{array}\right)+C\right] v= \\
\left.v^{T} A_{G} v=\begin{array}{l|l}
v^{T}\left[\begin{array}{l}
A_{G_{1}} \\
\hline \mathbf{0}_{(\mathbf{n}-\mathbf{i}) \times(\mathbf{i})}
\end{array}\right. & A_{G_{2}}
\end{array}\right] v \\
+v^{T} C v
\end{gathered}
$$

The first term at the right of the equality vanishes, since $A_{G_{1}} \mathbf{1}_{\mathbf{n}-\mathbf{i}}=0$ and $-A_{G_{2}} \mathbf{1}_{\mathbf{n}-\mathbf{i}}=0$. The second term can be easily evaluated, to obtain

$$
v^{T} A_{G} v=4
$$




\begin{tabular}{|c|c|c|c|}
\hline$\theta_{1}$ & $\bar{\theta}_{2}$ & Interconnection & $\theta$ \\
\hline stable & stable & phase & stable \\
stable & stable & counterphase & unstable \\
stable & unstable & phase & unstable \\
stable & unstable & counterphase & unstable \\
unstable & unstable & phase & unstable \\
unstable & unstable & counterphase & unstable \\
\hline
\end{tabular}

Table 1. Stability analysis of Theorem

4.1

Then, $A_{G}$ must have a positive eigenvalue and then the equilibrium $\bar{\theta}$ is unstable.

Now, we focus on the case with $\bar{\theta}_{1}$ or $\bar{\theta}_{2}$ unstable. We analyze the first case, since the other is similar. Then, $A_{G_{1}}$ has a positive eigenvalue with associated eigenvector $v_{1}$ such that

$$
v_{1}^{T} A_{G_{1}} v_{1}>0
$$

Define the vector

$$
v=\left[\begin{array}{c}
v_{1} \\
\alpha \mathbf{1}_{\mathbf{n}-\mathbf{i}}
\end{array}\right]
$$

with $\alpha$ chosen in a way that the components $i$ and $i+1$ of $v$ coincide. Then,

$$
v^{T} A_{G} v=v_{1}^{T} A_{G_{1}} v_{1}+\alpha^{2} \mathbf{1}_{\mathbf{n}-\mathbf{i}}^{T} A_{G_{2}} \mathbf{1}_{\mathbf{n}-\mathbf{i}}+v^{T} C v
$$

which actually is

$$
v^{T} A_{G} v=v_{1}^{T} A_{G_{1}} v_{1}>0
$$

Then, $\bar{\theta}$ is unstable.

Table 1 summarizes the results of Theorem 4.1. We are now ready to state and prove the main result of this article.

Theorem 4.2. Consider the graph $G$, with a bridge $e_{k}$ joining the nodes $v_{i}$ and $v_{i+1}$, and let $G_{1}$ and $G_{2}$ be the connected components of the graph $G \backslash\left\{e_{k}\right\}$. Then, $G_{1}$ and $G_{2}$ have the almost global synchronization property if and only if $G$ does.

Proof: First of all, let $\bar{\theta}=\left[\bar{\theta}_{1}^{T} \bar{\theta}_{2}^{T}\right]^{T}$ be an equilibrium point of $G$. According to theorem 4.1, $\bar{\theta}$ is stable only if $\bar{\theta}_{1}$ and $\bar{\theta}_{2}$ are too and the interconnection is in phase.

If $G_{1}$ and $G_{2}$ are a.g.s., the only locally stable set is the consensus, and due to the in phase interconnection, the only locally stable equilibria of $G$ is also the consensus and $G$ is a.g.s.

In the other direction, if $\bar{\theta}_{1}$ is a locally stable equilibrium of $G_{1}$, we chose $\bar{\theta}_{2}=\alpha \mathbf{1}$ such that the interconnection is in phase and we construct a stable equilibrium for $G$. Since $G$ is a.g.s., $\bar{\theta}$ must be a consensus equilibrium point.
Theorem 4.2 has many direct consequences. We point out some of them, with a brief hint of the respective proofs.

Proposition 4.4. If $G$ is a tree, it has the almost global synchronization property.

Observe that in a tree, every link is a bridge. The proof is based on the iterative application of Theorem 4.2. This result was previously proved in (Monzón and Paganini, 2006) using a different approach.

Corollary 4.1. If $G$ is a graph with the structure shown in figure 1, then $G$ is a.g.s. if and only if $G_{1}$ is.

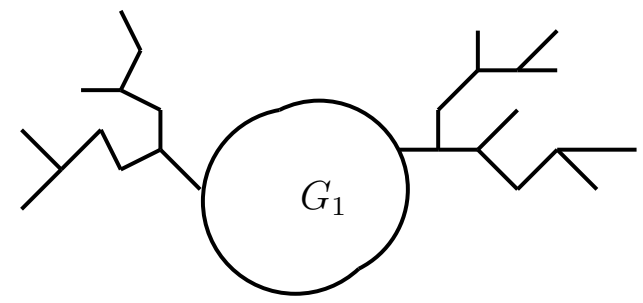

Figure 1. A graph with arboricities.

Corollary 4.2. If $G$ consists of two subgraphs $G_{1}$ and $G_{2}$ connected through a tree, as in figure 2, $G_{1}$ and $G_{2}$ are a.g.s. is and only if $G$ is.

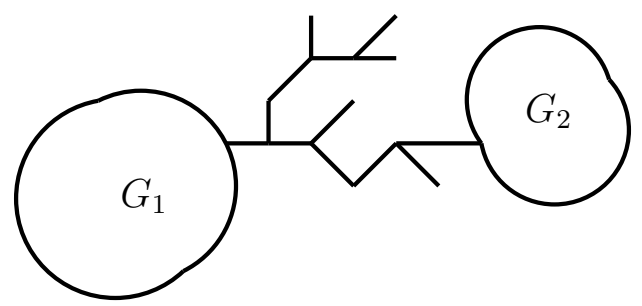

Figure 2. Two graphs connected by a tree

Summarizing, in the study of the a.g.s. property, every arboricity or interconnecting tree does not count.

Proposition 4.5. If $G$ is a tree and we build a new graph $K$ replacing every node of $G$ by an a.g.s. graph, then $K$ has the almost global synchronizing property.

The conclusion directly follows from the previous results. As an example, every vertex can be replaced by a complete graph, which always is a.g.s. (Monzón and Paganini, 2005). Observe that the interconnection can be done from any node of the a.g.s. graph. 
Example 4.1. (Moshtagh and Jadbabaie and Daniilidis, 2005) Consider $N$ agents $\left\{v_{1}, v_{2}, \ldots, v_{n}\right\}$ in the plane, that move around with constant unit velocity. The kinematic model of each agent is

$$
\begin{aligned}
\dot{x} & =\cos \left(\theta_{i}\right) \\
\dot{y} & =\sin \left(\theta_{i}\right) \\
\dot{\theta}_{i} & =\omega_{i}
\end{aligned}
$$

where $\omega_{i}$ is the control input. In this context, the synchronization of the agents a flocking state. An interconnection graph $G$ defines the interaction between agents. If we apply the control feedback

$$
\omega_{i}=\sum_{j \in \mathcal{N}_{i}} v_{j}^{T} X_{\theta_{i}}
$$

with $X_{\theta_{i}}=\left[-\sin \left(\theta_{i}\right), \cos \left(\theta_{i}\right)\right]^{T}$, the feedback systems has the following description:

$$
\dot{\theta}_{i}=\sum_{j \in \mathcal{N}_{i}} \sin \left(\theta_{j}-\theta_{i}\right)
$$

In order to see if the feedback control almost always leads to a flocking, it is enough to verify this property on the blocks of $G$. For example, if $G$ is a tree, we have flocking. Moreover, if we can design the interconnection, we can do it ensuring the property.

\section{CONCLUSIONS}

In this work we have studied how some algebraic properties of the underlying graph describing the interconnection of a symmetric Kuramoto model impose restrictions on the dynamical behavior. We focus on the particular case of the existence of a bridge between two agents. We proved that the interconnection by a bridge of almost global synchronized systems preserves that property. We think that the ideas we have presented here can be used to analyze other types of interconnection or graph interaction.

\section{REFERENCES}

Biggs, N. (1993). Algebraic Graph theory, Cambridge University Press.

Cvetkovic D., Doob M., Sachs H. (1979). Spectra of Graphs: Theory and Applications, Academic Press, Ney York.

Dussopt, L., Laheurte, J. (1999). Coupled oscillator array generating circular polarization, IEEE Microwave Guided Wave Letters 9(4): 160-162.

Ermentrout, G. (1985). The behavior of ring coupled oscillators, Journal of Mathematical Biology 23: 55-74.
Jadbababie, A., Lin, J., Morse, S. (2003). Coordination of groups of mobile autonomous agents using nearest neighbor rules, IEEE TAC 48(6): 988-1000.

Jadbabaie, A., Barahona, M., Motee, N. (2004). On the stability of the Kuramoto model of coupled nonlinear oscillators, Proc. of the $A C C$.

Khalil, H. (1996). Nonlinear Systems, PrenticeHall.

Kuramoto, Y. (1975). in International symposium on mathematical problems in theoretical physics, Lecture notes in Physics 39: 420, Springer.

Kuramoto, Y. (1984). Cooperative dynamics of oscillator community, Progress of Theoretical Physics Suppl (79): 223-240.

Marshal, J., Broucke, M., Francis, B. (2004). Formations of vehicles in cyclic pursuit, IEEE TAC 49(11): 1963-1974.

Monzón P., Paganini F. (2005). Global considerations on the Kuramoto model of sinusoidally coupled oscillators, Proc. of the 44th IEEE $C D C$ and the ECC, Sevilla, pp. 3923-3928.

Monzón P. (2006). Almost global stability of dynamical systems, Doctoral Thesis, Universidad de la República, Uruguay.

Monzón P., Paganini F. (2006). Global properties of symmetric coupled oscillators with non complete associated interconnection graph, Conresso Brasileiro de Automática, Bahia, Brazil, pp. 470-475.

Moshtagh N., Jadbabaie A., Daniilidis K. (2005). Distributed Geodesic Control Laws for Flocking of Nonholonomic Agents, Proc. of the 44th IEEE CDC and the ECC, Sevilla, pp. 28352838.

Rantzer, A. (2001). A dual to Lyapunov's stability theorem, Systems and Control Letters 42(3): 161-168.

Rogge, J. A., Aeyels, R. D. (2004). Stability of phase-locking solutions in a ring of unidirectionally coupled oscillators, Journal of Physics A: Mathematical and General 37: 11135-11148.

Strogatz S. (1994). Nonlinear Dynamics and Chaos: With Applications to Physics, Biology, Chemistry and Engineering, Perseus Book Publishing.

Strogatz, S. (2000). From Kuramoto to Crawford: exploring the onset of synchronization in populations of coupled nonlinear oscillators, Physica D (143): 1-20.

Winfree, A.T. (1980). The Geometry of Biological Time, Springer, New York.

York, R.A. (1993). Nonlinear Analysis of Phase Relationships in Quasi-Optical Oscillator Arrays, IEEE Trans. on Microwave Theory and Techniques 41(10): 1799-1808. 